\title{
The reliability and optimal data usage of BGAN Satellite Communications for Remote Outstations
}

\author{
Kinan Ghanem \\ Power Networks Demonstration Centre, \\ University of Strathclyde \\ Glasgow, UK \\ kinan.ghanem@strath.ac.uk
}

\author{
Federico Coffele \\ Power Networks Demonstration Centre, \\ University of Strathclyde \\ Glasgow, UK \\ federico.coffele@strath.ac.uk
}

\author{
James Irvine \\ Department of Electronic and Electrical \\ Engineering, University of strathclyde \\ Glasgow, UK \\ j.m.irvine@strath.ac.uk
}

\begin{abstract}
Telecommunications are a crucial element of smart grid technologies. Utility communication systems need to combine cost effectiveness with the need for high reliability. Due to its wide geographic coverage, satellite communication can be an appropriate alternative for power system automation in order to reach remote substations. This paper presents the experimental testing of Broadband Global Area Networks (BGAN) M2M technology between the supervisory control and data acquisition (SCADA) system running a primary front end processor (FEP) and the reclosers. Hardware test results for a number of different scenarios are discussed, compared and validated. The challenges for keeping the polls reliability at highest levels are also discussed.
\end{abstract}

\section{Keywords-Smart Grid, BGAN, SCADA, RTU}

\section{INTRODUCTION}

Telecommunications play significant role in enabling Distribution Networks Operators' (DNOs') to move closer towards the smart grid and achieve low carbon economy, but choosing the optimal communication technology can be problematic. Utility telecom system relies on different factors such as coverage, latency, and the DNOs' applications. Each communication technology has its own advantages and disadvantages for substation automation. In hard to reach areas, the construction and operation of telecommunications technologies such as fibre optics communications, other cables or mobile communications are expensive, and communications over satellite broadband global area networks become cost effective, while offering additional advantages of flexibility, robustness against natural disasters, and operational efficiency [1-3].

In power distribution networks, key metrics to be considered are bandwidth and the latency, as well as reliability and security. Satellite communication can be applied to exchange non-critical data and information between protection units, and control functions and may offer appropriate solutions for remote control and monitoring of substations. Advantages include availability and bandwidth combined with low deployment costs, while latency and the weather effects are the main drawbacks.

Satellite communications have been applied in power distribution networks with a focus on the use of Geo-stationary (GEO) satellites [6-8], whereas the use of Low Earth Orbit (LEO) satellites for remote meter reading and rural distribution automation is proposed in [9-10]. Very Small Aperture
Terminal (VSAT) satellite services have been applied for remote substation monitoring applications [10]. Satellite communication is also used for Global Positioning System (GPS) based time synchronization, which can offer microsecond accuracy in time synchronization [11]. Satellites can be also used as a backup for the existing substations communication networks and it can be used to route the data in case of link failure [12].

SCADA (Supervisory Control and Data Acquisition) control systems collect data from various sensors and distributed sites and transmit it to a unified computer for analysis, management and control. SCADA requirements vary based on the environmental conditions, the amount of information required to transmit, and the availability of power.

Utility companies use reclosers to isolate faults and re-route the power in an efficient manner. Reclosers require reliable connectivity for immediate control in cases of outage or disruption to transmission power lines. They can be controlled remotely by SCADA system and also provide data back to the operations centre, enabling power companies to prioritise restoration activities, minimise customer outage time and decrease any possible infrastructure damage[10]. These SCADA communications are generally low bandwidth and can be carried through many communication systems such as private telephone systems, fibre optic, existing radio mobile networks and satellite communications.

Satellite SCADA solutions such as BGAN M2M technology offer the promise of an easily integrated approach and IP-based connectivity service and could be suitable for managing remote assets such as reclosers of the power distribution networks.

In this work, a range of SCADA setups (mainly secondary front end processor (FEP) setup and Primary front end processor) is analysed to determine data usage and reliability. A secondary FEP setup is designed for the type of intermittent communications that are likely to be observed in the case of pole mounted reclosers (PMRs) and it requires to have dual endpoint capabilities. While the primary FEP does not require dual endpoint capable Remote Terminal Unite (RTU), it maintains a constant TCP connection between the FEP and RTU. In the remote SCADA via BGAN technology, Distributed Network Protocol (DNP3) is used for communication between control centers (using SCADA) and widely separated (remote) PMRs monitored and controlled by an RTU. 
This work aims to assess the data usage and reliability of broadband global area network (BGAN) machine to machine (M2M) communications for Interfacing SCADA with Power Mounted Reclosers (PMRs) via Remote terminal Unites (RTUs). Various tests were designed to determine the optimal data usage over a satellite network while taking into account the reliability requirements stipulated to be higher than $97.5 \%$ (The minimum acceptable reliability value under any circumstances). The paper provides results for data usage of different configurations and offers guidance on how the data usage could be optimised for different applications.

The rest of this paper is organised as follow: the setup and test case networks are explained in section 2; then the test results are presented section 3 , whereas, the optimal data usage is discussed in section 4, and finally, the conclusions about the results are discussed in section 5 .

\section{SETUP AND TEST CASE NETWORK}

The setup of end-to-end communications between the SCADA system PMRs, via the BGAN satellite communications using a primary Front End Processors (FEP) is executed, at the University of Strathclyde's the Power Network Demonstration Centre (PNDC). This includes the following installations:

- Installation of satellite units on the poles adjacent to the pole mounted reclosers;

- Full end-to-end reliable communications between the SCADA system running a primary front end processor (FEP) and the reclosers;

- End-to-end communication when using a secondary FEP.

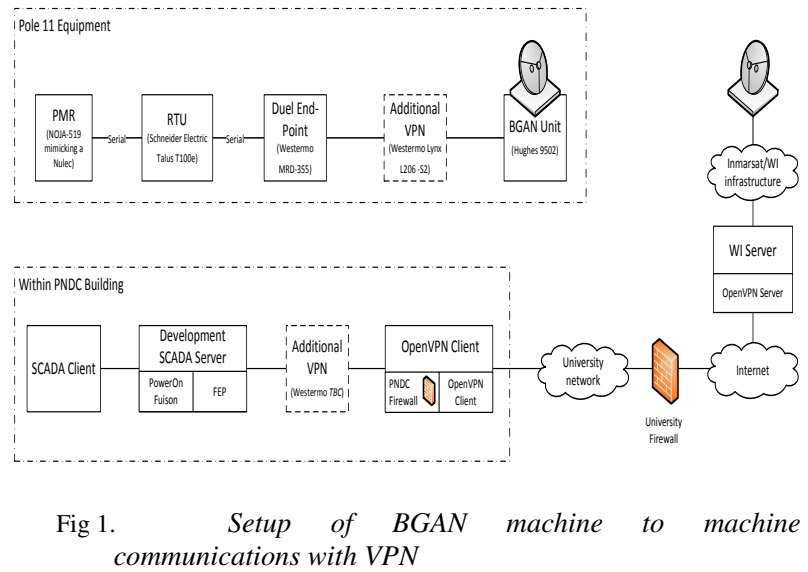

The test system explained in Fig. 1 included a Virtual Private Network (VPN) so that utility network communications are protected while passing through the third party network. However, while the VPN units were tested using a direct link, the tests via the satellite network did not use the VPN due to an issue with the power budget for the VPN unit on the pole mounted equipment. The VPN will not add significantly to the traffic over the satellite network.
The data usage is determined using Wireshark, and it is verified against the data usage recorded via the Wireless Innovation (WI) portal which provides the satellite network's view of the data traffic. Good and bad poll rates will be used to determine the number of failed polls. Where required, the FEP $\log$ and tester's notes will be used to determine the number of failed controls or failed reports of unsolicited changes.

\section{TEST RESULTS:}

The data optimisation when testing polled analogues was carried out in accordance with the following requirements (Analogues Polling, Digitals Unsolicited, Time download once in 24 hour period). Three tests were completed and configured to achieve the following:

- Test 1 aims to validate of data from Wireshark with the data from the WI portal.

- Test 2 - aims to study the changes in heartbeat frequency and RTU timeout during the test.

- Test 3 - aims to study the changes in polling frequency and RTU timeout during the test.

All the tests have polled analogues $\mathrm{x} 4$, unsolicited digitals and a time synchronisation. The duration of each test varies from 4-17 hours. For each case the recorded results have been extrapolated to give a representation of 31 days usage, to give a better estimation of monthly cost in terms of data allowance restrictions.

\section{A. Test 1 - configurations:}

The primary purpose of this test is to compare and validate the results from the satellite operator with the results obtained from Wireshark locally.

Two different RTUs are used in the test, and, the polling period is selected to be 5 minutes and 30 minutes respectively. The socket heartbeat time is chosen to be 4 minutes for both RTUs and TCP time out is 8 minutes. The testing period lasts for 4 hours. The associated SCADA configurations for test 1 are shown in Table 1.

TABLE I. SCADA CONFIGURATION OF TEST_1

\begin{tabular}{|c|c|}
\hline Attribute $=$ Value in sec & Description \\
\hline Disable Timesync $=2$ & $\begin{array}{l}\text { FEP will only send a time synchronization } \\
\text { if the RTU requests. }\end{array}$ \\
\hline Do Network Time $=1$ & Download time using TCP/IP messages \\
\hline Do Delay Measure $=0$ & Do not perform delay measure (radio only) \\
\hline Time Download $=86400$ & Download time to RTU only every $24 \mathrm{~h}$ \\
\hline RTU10 App Timeout $=10$ & $\begin{array}{l}\text { Timeout used by the FEP when waiting for } \\
\text { an app. message to come back from the } \\
\text { RTU10. }\end{array}$ \\
\hline RTU9 App Timeout $=10$ & $\begin{array}{l}\text { Timeout used by the FEP when waiting for } \\
\text { message to come back from the RTU9. }\end{array}$ \\
\hline
\end{tabular}




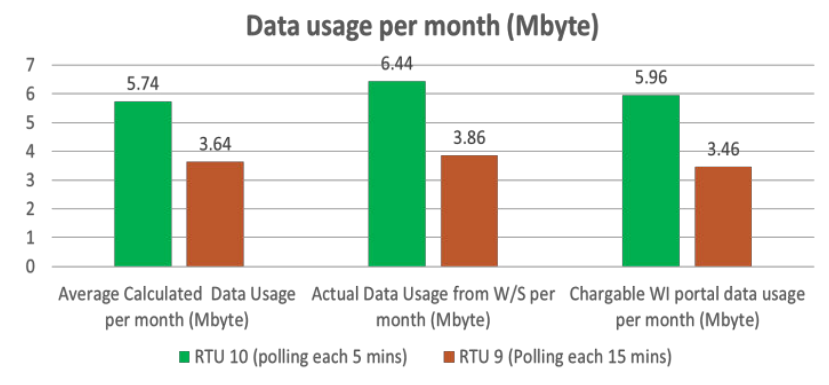

Fig 2. Monthly data usage (in Mbyte) for Test 1

Results from Test 1 shows that the monthly data usage of both tested RTUs meet our calculated data usage. The calculated data correlates to within $5 \%$ of the chargeable data as reported from the satellite operator (WI) as it is illustrated in Fig. 2.

\section{B. Test 2 - configurations:}

Test 2 aims to determine by how much socket heartbeats can be reduced in relation to the analogue polling. In this test, the timeout of the RTU10 is selected to be 15 seconds, whereas the RTU9 timeout is 10 seconds. The polling period of RTU10 and RTU9 are selected to be 5 minutes and 10 minutes respectively, whereas the socket heartbeat time is chosen to be 4 minutes for RTU10 and 960 minutes for RTU9. TCP time out is chosen to be 8 mins for RTU10 and 20 mins for RTU9. The testing period for Test 2 is 17.25 hours. SCADA configurations for Test_2 have the following differences from Test_1:

- RTU10 timeout changed from 10 seconds to 15 Seconds

- $\quad$ TCP time out of RTU9 changed from 8 minutes to 20 minutes.

- $\quad$ SKT H/B Time of RTU9 changed from 4 minutes to 960 minutes.

- Test_2 period time is $\mathbf{1 7 . 2 5}$ hours

Fig. 3 presents monthly data usage for both RTU 10 and RTU 9. The chargeable data usage measured by WI portal is closely matched by both the average projected data and the captured data from Wireshark for RTU10 (which has high success polls rate comparing to the RTU9 as we it can be shown later on polls reliability).

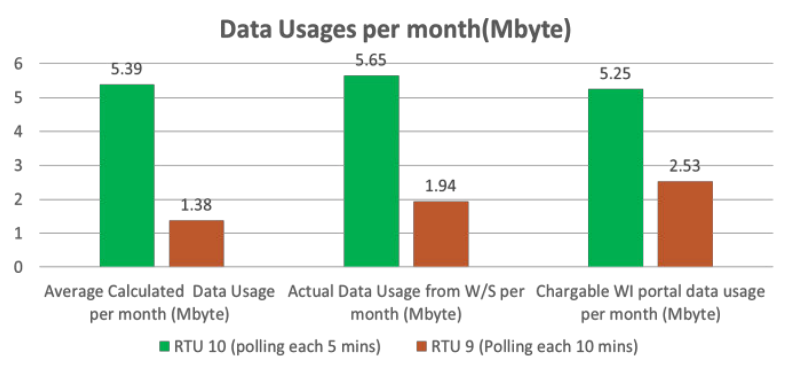

Fig 3. Monthly data usage (in Mbyte) for Test_2
Obtained results from Test 2 show the following:

- Wi Portal data usage matched our results for RTU10 (with timeout=15 seconds).

- Increasing of polling period can decrease the monthly data usage and keep the reliability at acceptable level. The heartbeat interval time has also been increased from 4 minutes to 960 minutes, which is $16 \mathrm{hrs}$ on a $17.5 \mathrm{hr}$ test duration. so about 260 fewer heartbeats and associated data.

- RTU10 with timeout 15 seconds gave more reliable polls success rate $(94.2 \%$ in the previous test as it will be shown in poll reliability subsection 4.2).

- The majority of bad polls are due to RTU timeout (as RTU timeout is increased RTU10 returned a better poll success rate).

\section{Test 3 - configurations:}

Test 3 determines how much socket heartbeats can be reduced in relation to the analogue polling and checks the effects of increasing the RTU time out on the polls reliability. In Test 3, the timeout of the RTU10 and RTU9 are selected to be 15 seconds. The polling period of RTU10 and RTU9 are selected to be 5 minutes and 10 minutes respectively, whereas, the socket heartbeat time is chosen to be 4 minutes for RTU10 and 960 minutes for RTU9. TCP time out is chosen to be 8 mins for RTU10 and 20 mins for RTU9. The testing period in Test_3 lasts for 8 hours. SCADA configurations for Test_3 have the following differences from Test_2:

- RTU10 timeout changed from 10 seconds to 15 Seconds

- $\quad$ TCP time out of RTU9 changed from 8 minutes to 20 minutes.

- $\quad$ SKT H/B Time of RTU9 changed from 4 minutes to 960 minutes.

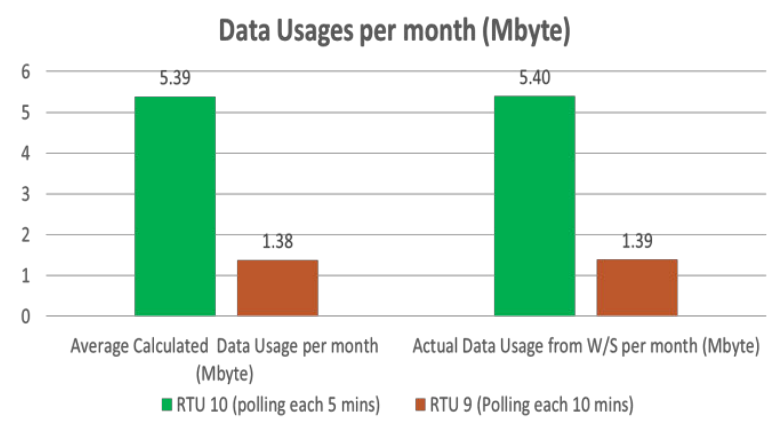

Fig 4. Monthly data usage (in Mbyte) for Test_3

Illustrated Results in Fig. 4 show a significant reduction in data usage for a polling rate of 10 minutes and socket heartbeat 
of 960 minutes while keeping the reliability at acceptable threshold. Monthly data usage for RTU 9 with 10 mins polling period (about 1.4 Mbyte) doesn't exceed the DNO's monthly data usage threshold (1.8 Mbyte a month) and is a good optione. Moreover, the socket heartbeat can be reduced without any effects of the polling reliability due to the frequency of requested polls from SCADA.

\section{The reliability of the Primary FEP Testing:}

The primary FEP testing seeks to characterise the typical reliability (bad polls rate) and polls reliability has been analysed for every test. The reasons beyond having bad Polls in our previous tests are identifying based on correlating of both the FEP and Wireshark logs.

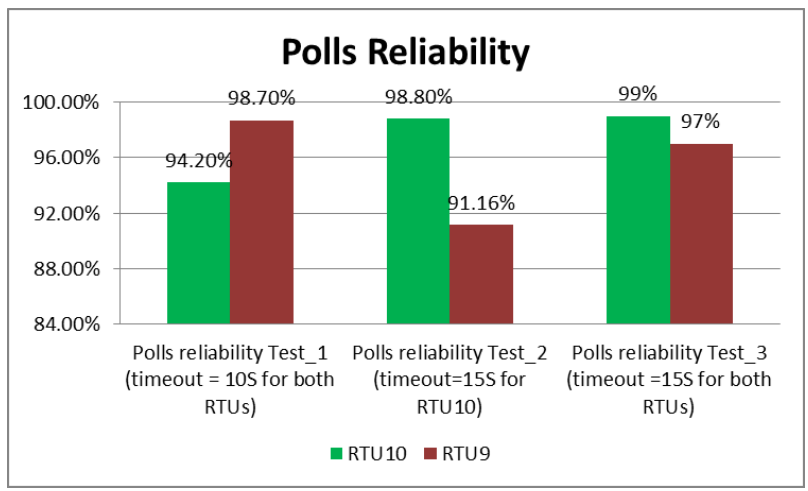

Fig 5. Polls reliability for each test

Polls reliability checks for the different tests in Fig.5 illustrate the following:

- Most bad polls are caused by RTU timeout (10 seconds in this test).

- RTU (10) with a timeout of 15 seconds returned a higher poll success rate than in previous test, Test $1(94.2 \%)$ with timeout of 10 seconds.

- Increasing RTU timeout can increase the polls success rate(Test 3)

- Weather condition changed between tests and that affected the results

\section{OPTIMAL DATA USAGE}

Based on the previous tests, optimal monthly data usage can be estimated for different applications and scenarios. Analogue polling periods can be configured taking into consideration that reliability should be kept at a high level. According to the results, the socket heartbeat period can be increased without any effects on polling reliability which decreases the monthly data usage. For digital unsolicited application where the events come from the terminal rather than from the SCADA FEP, the unsolicited digital period can be estimated and then it can be used to calculate the data usage based on the results we obtained from the previous test.
The results of the testing have demonstrated that the primary FEP is the best option to achieve optimal data usage and reliability. Secondary FEP was also tested and the results demonstrated that the data usage is significantly higher than Primary FEP. Analysis results of secondary FEP based on captured data from Wireshark and obtained data from the satellite operator illustrates that the average data usage for one background poll is 1342 Bytes, whereas the data usage of an analogue poll in primary FEP is 282 Bytes and each heartbeat requires 248 bytes. This means that the message exchange size in the secondary FEP is 4.78 times higher than that in the primary FEP (when polling only analogue). This will enable the primary FEP to be the best option to achieve the lowest optimal data usage and at the same time keep the reliability of the polls at highest levels.

The capture data from Wireshark slightly differs from the recorded data rates from the satellite operator. The difference is due to retransmissions of packets due to errors or lost acknowledgements, and also due to the firewall in the WI gateway.

The heartbeat rate (governed by the 'socket heartbeat' parameter) will affect the results and so testing involves a range of different heartbeat rates, in an attempt to determine information about the compromises between data cost and the reliability of the link. Further work is required in order to investigate what is causing the bad polls at low heartbeat rates. Identifying the source and potential solutions may be the key to getting reliable communications at low data usage when using a primary FEP.

\section{CONCLUSIONS AND FUTURE WORK}

Satellite SCADA solutions that provide global coverage in insulated areas can be used in the power utility networks to control and manage of substations and remote assets. This paper investigated the reliability of BGAN M2M technology in terms of connectivity and cost effectiveness when it is applied in the power companies, and provided a summary of the testing results for data usage of different configurations and offered guidance on how the data usage could be optimized. Various tests were designed to determine the optimal data usage over a satellite network while taking into account the reliability requirements stipulated to be higher than $97.5 \%$. Results show that the reliability of the connection can be maintained and the optimal data usage for communications over BGAN that interface SCADA with key network assets via RTUs can be achieved. The main finding of the experimental tests can be summarized as follow:

- The importance of good success poll rates when considering the data usage. As low data usage for any setup or configuration should not be considered until assuring that the success poll rate is exceeding the minimum acceptable threshold of the success poll rate.

- The primary FEP is found to be the best option to achieve the lowest optimal data usage and at the same time keep the reliability of the polls at highest levels. 
- $\quad$ RTU timeout plays significant role in increasing the polls reliability. This is in relation to an increase from 10 seconds to 15 seconds and a potential explanation could be due to the effect of changeable weather conditions on satellite communications.

Future work may further investigate the effects of severe weather condition on the reliability of the BGAN satellite communications technology link.

\section{ACKNOWLEDGMENTS}

This project was funded under the noncore research programme contributed by Scottish and Southern Energy Networks (SSEN) of the University of Strathclyde's PNDC (http://pndc.co.uk/). we were working with engineers from SSE, who they supported us and helped delivering such a successful project.

\section{REFERENCES}

[1] Holbert K. E., Heydt G. T., and Ni H., "Use of satellite technologies for power system measurements, command and control," Proc. the IEEE, vol. 93, no. 5, pp. 947 - 955, May 2005.

[2] Ekici E, Akyildiz IF, and Bender MD. "A distributed routing algorithm for datagram traffic in LEO satellite networks." IEEE/ACM Trans. Netw., vol. 9, no. 2, pp.137-147, April 2001.

[3] Vaccaro A. and Villacci D., "Performance analysis of low earth orbit satellites for power system communication," Electric Power Systems Research, vol. 73, no. 3, pp. 287-294, 2005.

[4] Xiao, X., Fu, Z., Liu, G. and Deng, C., 2010, October. A backup data network for power system automations based on satellite communication. In Power System Technology (POWERCON), 2010 International Conference on (pp. 1-5). IEEE.

[5] Madani, V., Vaccaro, A., Villacci, D. and King, R.L., 2007, August. Satellite based communication network for large scale power system applications. In Bulk Power System Dynamics and Control-VII. Revitalizing Operational Reliability, 2007 iREP Symposium (pp. 1-7). IEEE.

[6] Murty Y. S. N., "Hybrid communication networks for power utilities," Proc. The Power Quality, pp.239-242, Jun. 1998.

[7] Holbert K. E., G. T. Heydt, and H. Ni, "Use of satellite technologies for power system measurements, command and control," Proc. the IEEE, vol. 93, no. 5, pp. 947 - 955, May 2005.

[8] Marihart D. J., "Communication Technology Guidelines for EMS/SCADA systems,” IEEE Trans. Power Deli., vol. 16, no. 2, pp. 181-188, April 2001.

[9] Ekici E, Akyildiz IF, and Bender MD. "A distributed routing algorithm for datagram traffic in LEO satellite networks." IEEE/ACM Trans. Netw., vol. 9, no. 2, pp.137-147, April 2001.

[10] Dhar T. and Tang T., "Present and future communication technologies for distribution automation in rural Queensland, Australia," Proc. Int. Conf. Energy management and power Delivery, pp.650-655, Mar. 1998.

[11] Yang, Q., 2012, June. Satellite based "Power Utility Intranet" for smart management of electric distribution networks: The AuRA-NMS case study. In Communications (ICC), 2012 IEEE International Conference on (pp. 2822-2826). IEEE.

[12] Qureshi, M., Raza, A., Kumar, D., Kim, S.S., Song, U.S., Park, M.W., Jang, H.S., Yang, H.S. and Park, B.S., 2008, April. A survey of communication network paradigms for substation automation. In Power Line Communications and Its Applications, 2008. ISPLC 2008. IEEE International Symposium on (pp. 310-315). IEEE. 